\title{
Combined Therapy with Rosiglitazone, Rofecoxib and Celecoxib Enhances Cardiovascular Side Effects through Cytochrome P450 Pathway of Arachidonic Acid Metabolism in Diabetic Arthritis Model Rats
}

\author{
Xuya Zhou ${ }^{1}$, Ying Liu ${ }^{1}$, Deqin $\mathrm{Xu}^{1}$, Jie Bao ${ }^{1}$, Yaru $\mathrm{Cao}^{2}$ and Yong $\mathrm{Jin}^{3 *}$ \\ ${ }^{1}$ Department of Pharmacy, Hospital of Armed Police of Anhui Province, Hefei 230061, \\ China \\ ${ }^{2}$ Department of Pharmacy, The Fourth People Hospital of Wuhu, Wuhu 24000,China \\ ${ }^{3}$ School of Pharmacy, Anhui Medical University, Hefei 230032, China
}

Xuya Zhou and Ying Liu contributed equally to this work and should be considered co-first authors.

\begin{abstract}
A B S T R A C T
Many drugs increase the risk of cardiovascular disease, such as thiazolidine diketone: rosiglitazone, selective cyclooxygenase (COX)-2 inhibitors: rofecoxib, celecoxib. This study was aimed to find out whether unification of two drugs would increase cardiovascular side effects. Type 2 diabetic rat model was induced through feeding with fodder containing high sugar and lipid and intraperitoneal injection of streptozotocin. The adjuvant-induced arthritis model was established by Paraffin/BCG. We used western blot to explore the protein expression levels of COX-2 and cytochrome P450 (CYP) 4A1. The expression of CYP4A1 was detected by immunohistochemistry. Enzyme-linked immunosorbent assay (ELISA) was used to detect the prostaglandin E2 (PGE-2), 20-Hydroxyeicosatetraenoic acid (20-HETE), endothelin 1 (ET-1) and B-type natriuretic peptide (BNP) in blood serum of diabetes complicating arthritis rats. Blood pressure was measured by a noninvasive caudal artery blood pressure meter. Western blot analysis showed that unification of two drugs caused protein expression levels of COX-2 to reduce obviously and increased protein expression levels of CYP4A1. Immunohistochemistry showed the increased expression of CYP4A1. ELISA indicated that the decreased production of PGE-2 and the increased production of 20-HETE, ET-1 and BNP in blood serum. The blood pressure of rats was increased. The results indicated that unification of two drugs could induce CYP4A1 and inhibit COX-2, and increased production of 20HETE from arachidonic acid (AA) metabolism. The increased 20-HETE led to the hypertension which could increase markers (ET-1 and BNP) of cardiovascular disease in diabetes complicating arthritis rats. The results showed that the combination of the two drugs could increase the cardiovascular side effects, mainly through the arachidonic acid metabolic pathway CYP450.
\end{abstract}

\begin{tabular}{l} 
Article Information \\
Received 19 August 2019 \\
Revised 21 December 2019 \\
Accepted 14 March 2020 \\
Available online 12 January 2021 \\
Authors' Contribution \\
\hline XZ and YJ designed the study. XZ and \\
YL performed experimental work and \\
analyzed the data. XZ, YL, DX, JB \\
and YC performed the experiments. \\
Key words \\
COX-2 inhibitors, Arthritis, \\
Thiazolidinedione, Diabetes, CYP4A1, \\
20-HETE
\end{tabular}

\section{INTRODUCTION}

$\mathrm{D}$ iabetes mellitus (DM) causes the accumulation of hyperglycemia and the progression of DM is a set of alterations that alterations is directly associated with pancreatic $\beta$-cell loss of function (Ralph, 2009). Epidemiological studies showed that the incidence of $\mathrm{DM}$ is rising at pandemic levels all over the world, with more than 300 million people estimated to be affected by the year of 2025 (Desk et al., 2002). As a kind of chronic multisystem autoimmune disease, rheumatoid arthritis(RA) is characterized by persistent inflammation (Bax, 2001).

\footnotetext{
* Corresponding author: jinyong@ahmu.edu.cn 0030-9923/2021/0002-0483 \$ 9.00/0

Copyright 2021 Zoological Society of Pakistan
}

It is a stubborn autoimmune disease that affects $0.5 \%$ to $1 \%$ of the global general population (Barton, 2009). Some reports and data from registries also confirm that the morbidity of type 2 diabetes mellitus (T2DM) is increased in RA patients (Roberto et al., 2016). Theoretically, combined therapy with antidiabetic and anti-inflammatory should be common, but relevant information is rare reported.

Thiazolidinediones a class of drug, that provided a main breakthrough in the control of type 2 diabetes since 1990 (Yasmin and Jayaprakash, 2017). Rosiglitazone is one of the peroxisome activated receptor (PPARs) activated by thiazolidone dione and is also a ligandactivated transcription factor being a member of the nuclear hormone receptors superfamily, which has shown good application prospects in patients with diabetes. Because of its insulin-sensitizing effects, T2DM that is 
uncontrolled by insulin (Varga et al., 2001). More than 33,000 diabetics enrolled in 35 health plans in the United States of a retrospective cohort study, in this study, patients taking TZDS were diagnosed with heart failure more frequently than patients taking other oral hypoglycemic drugs (Delea et al., 2004). It was also announced on September 23 by the US Food and Drug Administration (FDA) and the European Medicines Agency (EMA), that is, the FDA intends to impose heavy restrictions on the application of rosiglitazone, a drug that is now almost available for clinical practice. Notably, the FDA imposed restrictions on the prescription and dispensing of rosiglitazone in 2010 because of concerns about its cardiovascular safety (Alzaid, 2010). The restriction was removed because an independent reassessment of the recording trial found no significant difference between rosiglitazone and sulfonylurea/metformin take a risk of stroke, cardiovascular (or inscrutability cause) death, or myocardial infarction (Kenneth et al., 2013).

Anti-inflammatory drug selective cyclooxygenase-2 (COX-2) inhibitors act as an effective anti-inflammatory effect and have used as an important option which used to improve rheumatoid arthritis (Sundy, 2001). Merck, the manufacturer of refecoxib, withdrew his product from the worldwide market on September 30, 2004, because arthritis patients taking rofecoxib had a higher danger of adverse cardiovascular events and wind-stroke syndrome than those taking naproxen, and cardiovascular problems associated with high doses of other coenzyme agents such as diacoxib and celecoxib in addition (Mukherjee et al., 2001; Ray et al., 2004). Currently, celecoxib (Celebrex), which is less potent than rofecoxib, is the only coxib on the market (Zhang et al., 2014). Cox-2 inhibitors can reduce the production of platelet aggregation inhibitor prostacyclin I2 (PGI2) and have a small effect on the production of the potent platelet activator thrombin A2 (TX) (FitzGerald, 2004; Cheng, 2002), and the cardiovascular problems may be caused by an overly of thromboxane A2.

From the foregoing, these two kinds of drugs both have the potential risk in aggravating cardiovascular disease. By observing the mechanisms of TZD and coxibs, we found that the side effects of TZD may originate from the metabolite, 20-HETE. Possible mechanism about the side effects of coxibs may result from the breaking down of the PGI2/TXA2. In this case, we image that since these two types of drugs all have cardiovascular adverse reactions, and what would be happening if combination of these two types of drugs? Occasionally, we found that they all partly participate in the metabolism of the arachidonic acid (AA). As a $\omega-6$ polyunsaturated fatty acids, AA was found to bind to cell membranes. It remained esterified to the sn-2 position of glycerolipid, but the release rate of the unesterified form was lower. In response to stimulation, phospholipase $\mathrm{A} 2$ releases unesterified $\mathrm{AA}$ and metabolizes it into bioactive metabolites known as eicosomes (Imig, 2012). AA is known to be widespread in eukaryotic cells, and cycloperoxidase and lipid oxidase to produce prostaglandin (PGs) and icosecanoic acid peroxide leading catalysis of its metabolism. and its metabolism is catalyzed by cycloxygenases and lipoxygenases to produce PGs and hydroperoxyeicosatetraenoic HPETE acid (Weerasinghe et al., 2004). Recently the third pathways of arachidonic acid metabolism confirmed is metabolic pathway of cytochrome P-450 dependent monoxygenases, which include hydroxylases and epoxygenases. The synthesis of EETs from AA catalyzed by cyclooxygenase. The hydroxylase metabolize AA to 19,20-hydroxyeicosatetraenoic acid (19,20-HETH). In brief, the new pathways has shown an important role in the pathogenesis of hypertenion (Kroetz and Zeldin, 2002). 20-HETE is a vasoconstrictor metabolite of arachidonic acid formed in vascular smooth muscle (VSM) cells through the action of cytochrome P450 $\omega$-hydroxylase 4A (CYP4A) (Lukaszewicz et al., 2013), and 20-hydroxy dodecaenoic acid is an effective substance to regulate vascular tension (Nithipatikom et al., 2001). EET is a powerful vasodilator, especially in arterioles (Harder et al., 1995), they acting on the vascular smooth muscle and unfold calcium-sensitized potassium $(\mathrm{KCa})$ channels, leading to membrane vasodilation and hyperpolarization (Gebremedhin et al., 1992; Pfister and Campbell, 1992; Campbell et al., 1996). EETs also has powerful anti-inflammatory properties in the vascular system by inhibiting the activation of NF-kB (Lukaszewicz et al., 2013). However, the vascular function of production of 20-HETE by the action of CYP 4A has been less well studied. It has been gradually research and discovery in nearly ten years that 20-HETE. Studies have shown that it stimulates endothelial dysfunction and activation, increases the contraction and proliferation of smooth muscle and leading to hypertension (Kroetz and Zeldin, 2002), it may bring some research prospects in the future. It was reported that Coxibs inhibits the AA pathway of COX (Reddy et al., 2015), thus indirectly enhancing the other metabolic pathways. TZD can induce CYP4A to increase the level of 20-HETE of the CYP pathway (Juge-Aubry et al., 1997). Therefore, it has theoretical possibility that drugs combination by TZD and coxibs increase cardiovascular adverse reaction. In this study, we investigated whether concurrent combination therapy of anti-inflammatory (rofecoxib, celecoxib) and antidiabetic rosiglitazone would exert a more severe impairment effect in cardiovascular serious side effects via diabetes complicating arthritis in a rodent model. For this, we attempt to explore the biological mechanisms in deeper level. 


\section{MATERIALS AND METHODS}

\section{Animals}

The male Sprague-Dawley rats with the weight ranging 180-220g were acquired from the Laboratory Animal Center of Anhui Medical University. In the experiment we placed rats in cages with constant temperature $\left(20-25^{\circ} \mathrm{C}\right)$, humidity control (40\%-70\%), and daily light $12 \mathrm{~h}$-dark light $12 \mathrm{~h}$. In addition, experimental animals were used under the Institutional Animal Ethics Committee and Animal Care Guidelines of Anhui Medical University. Before the experiment, for the rats, food was forbidden for $12 \mathrm{~h}$ but not water. Animal experiments shall be conducted in accordance with the regulations on feeding and using experimental animals issued by the Committee of Ethics of Animal Experimentation of Anhui Medical University (Hefei, China). The rats were put into 8 groups by random division (control group, diabetes model group, combined disease model group, rosiglitazone group, rolacoxib group, celecoxib group, treated with rosiglitazone and rofecoxib group, treated with rosiglitazone and celecoxib group, $n=8$ rats per group).

\section{Reagents}

STZ, rofecoxib, celecoxib and rosiglitazone were purchased from Sigma. The blood glucose meter was purchased from Novo Nordisk. Rabbit anti-CYP4A, anti-COX-2 and anti-actin monoclonal antibodies were purchased from Cell Signaling Technology. The ELISA kits for PGE2, 20-HETE, ET-1 and BNP were purchased from $\mathrm{R}$ and $\mathrm{D}$ Systems. All chemical reagents are of analytical grade or above.

\section{Model}

Male rats with insulin resistance (IR) was induced by fed with high-sugar and high-lipid fodder (contained $68 \%$ basic feed and $10 \%$ yolk and $6 \%$ lard and $15 \%$ sugar) for 6 weeks. After that, for the rats, food was forbidden for $12 \mathrm{~h}$ but not water. And diabetes model was induced with a singer intraperitoneal injection of streptozotocin. The adjuvant-induced arthritis model was established by Paraffin/BCG. All experiments were performed in accordance with the guidelines for animal research established by the guidelines of the ethics committee of Anhui Medical University (Certificate number: 20170282).

After creating the DM models of rats, rosiglitazone was continuously by gavage for 2 weeks; and after adjuvantinduced arthritis model was established, rofecoxib or celecoxib was delivered by gavage. Drug combinations will be used in diabetes complicating arthritis rats.

\section{Mean arterial pressure (MAP) measurements}

We monitor blood pressure in conscious animals. Tail blood volume was measured using volume-pressure recording sensors and capping sleeve. (Anhui Zhenghua Biological Equipment Co., Ltd.), we measured blood pressure noninvasively once a week. In order to obtain an average of 10 stable measurements, blood pressure was measured multiple times in a single rat. The results are shown as the average MAP value of a single rat.

\section{ELISA analysis}

Serum levels of PGE-2, 20-HETE, ET-1 and BNP were determined by ELISA. The stop solution had an effect on the serum sample, changing it from blue to yellow, and the intensity of the color was measured at $450 \mathrm{~nm}$ by adopting a spectrophotometer. To determine the concentration of PGE-2, ET-1 and 20-HETE in the sample, the optical density of the sample was put into comparison with the standard curve. If the concentration of the sample goes beyond the limit of the standard curve, another dilution and check is conducted.

\section{Western blot analysis}

The protein extraction kit (BestBio, institute of biotechnology, Shanghai, China) was used to extract protein from liver tissue. The BCA assay kit (BestBio) was used to determine the protein level. After SDS-PAGE separation, the protein $(50 \mathrm{mg})$ from the sample was transferred to PVDF membrane and incubated with TBST containing $5 \%$ skim milk for about $2 \mathrm{~h}$ at $37^{\circ} \mathrm{C}$. After that, PVDF membrane and specific primary antibody were incubated overnight at $4^{\circ} \mathrm{C}$. After that, anti-cox-2 and p65 monoclonal antibodies of the great mouse were diluted to 1:400, and anti-actin was added at a ratio of 1:500. On the second day, the culture membrane was rinsed 3 times with TBS-Tween $20(0.075 \%)$ and then incubated at $37^{\circ} \mathrm{C}$ for about $1 \mathrm{~h}$ with horseradish peroxidase combined with rabbit and mouse anti-antibodies $(1: 10,000)$. Then wash the membrane three more times. The protein was observed with the chemiluminescence kit (ECL).

\section{Immunohistochemistry methods}

Liver, kidney and heart tissues were fixed in $10 \%$ neutral buffer formalin solution, which were embedded in paraffin, and stained with conventional histology. To obtain antigen retrieval, partially dewaxed in xylene, dehydrated citrate in alcohol and microwave for 15 minutes. Inhibit endogenous peroxidase activity, partially deparaffinized, and then use $0.3 \%$ hydrogen peroxide for 15 minutes. All parts were further incubated with $2 \%$ bovine serum albumin and primary antibody CYP4A (administered) at $16 \mathrm{~h}$ at $4^{\circ} \mathrm{C}$. After rinsing, sections were incubated in 
biotinylated secondary antibody at room temperature for 60min. The expression of CYP4A was observed by staining with 3, 3-diaminobenzidine tetrachloric acid (DAB). The sections were dehydrated after hematoxylin restaining and observed after CYP4A positive area was placed in the fibrosis area. Each liver section was quantitatively analyzed from five domains.

\section{Statistical analysis}

Univariate analysis of variance (ANOVA) and LSD multiple comparison test were used for statistical analysis. SPSS 17.0 statistical software was used (SPSS, Chicago, Illinois, USA). The data provided are the mean \pm standard deviation of three independent experiments.

\section{RESULTS}

The associated index about diabetes and arthritis.

As shown in Figure 1, we measured the blood glucose to make sure that diabetes models were well established by induced with a singer intraperitoneal injection of streptozotocin between control group and model group. As shown in Figure 2, the adjuvant-induced arthritis model was established by Paraffin/BCG.

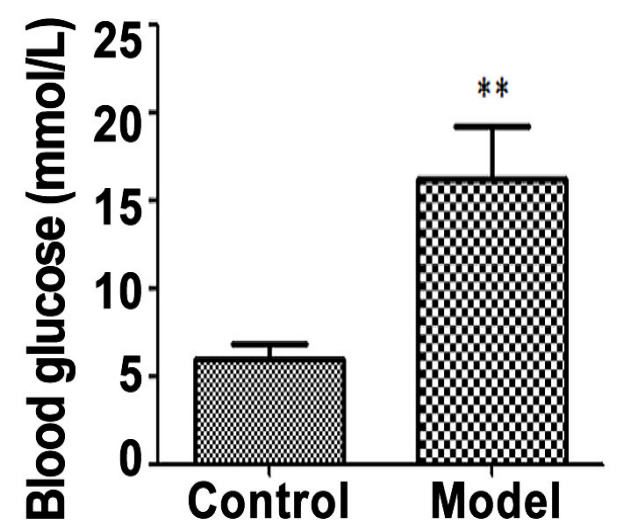

Fig. 1. Blood glucose level of control, diabetes and arthritis model rat.

Effect of combination medication treatment on mean arterial pressure (MAP).

To study the influence of unification medication treatment on blood pressure in diabetes complicating acute inflammation rats, we measured blood pressure once a week in all groups. Before the medication treatment, blood pressure was measured, which showed that there was no significance difference among different groups and the levels of blood pressure among all groups was within a normal range. As shown in Table I, the results indicated that there were no significant difference between them.

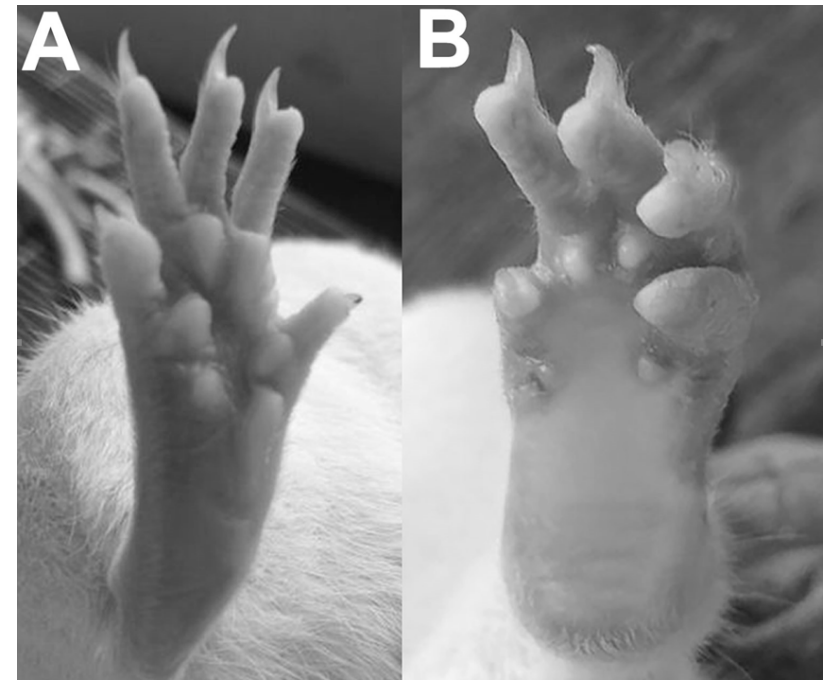

Fig. 2. Macroscopic observation on paw swelling of rats. A, Control group rats; B, Model (Adjuvant-induced arthritis) group rats.

Effect of combination medication on PGE-2 in the blood serum of rats

ELISA was used to determine the effects of combination medication on the production of PGE-2 to confirm that the COX-2 pathway has been blocked. As shown in Figure 3A, by comparing the data with the control group, PGE-2 levels in the model group climbed greatly owing to inflammation. The third group of model group of combined disease showed significantly increase in the production of PGE-2 $(\mathrm{P}<0.01)$. The rosiglitazone-treated group displayed signs of improvement. Administration of rofecoxib or celecoxib reduced the production of PGE$2(P<0.05)$, the production of PGE-2 was significantly decreased in the combination medication group compared with the model group $(P<0.01)$.

Effect of combination medication on 20-HETE in the rat blood serum

As shown in Figure 3B, ELISA was used to evaluate 20-HETE in the blood serum of the model group. 20-HETE was significantly increased in comparison with the control group $(\mathrm{P}<0.05)$. By comparing the data with model group, each treatment group increasing the levels of 20HETE. Notably, the combination medication remarkably increased the 20-HETE levels $(P<0.01)$.

Effect of combination medication on ET-1 in the blood serum of rats

The concentration of ET-1 in rat serum is shown in Figure 3C. It shows that the model group displayed increased levels of ET-1 compared with the control 
Table I. Effects of drug-combination on the tail arterial blood pressure in the experimental groups.

\begin{tabular}{lllllllll}
\hline Group No. & $\mathbf{1}$ & $\mathbf{2}$ & $\mathbf{3}$ & $\mathbf{4}$ & $\mathbf{5}$ & $\mathbf{6}$ & $\mathbf{7}$ & $\mathbf{8}$ \\
\hline Blood pressure (mmHg) & 86.682 & 92.069 & 96.398 & 105.453 & 101.19 & 103.272 & 127.396 & 119.233 \\
\hline
\end{tabular}
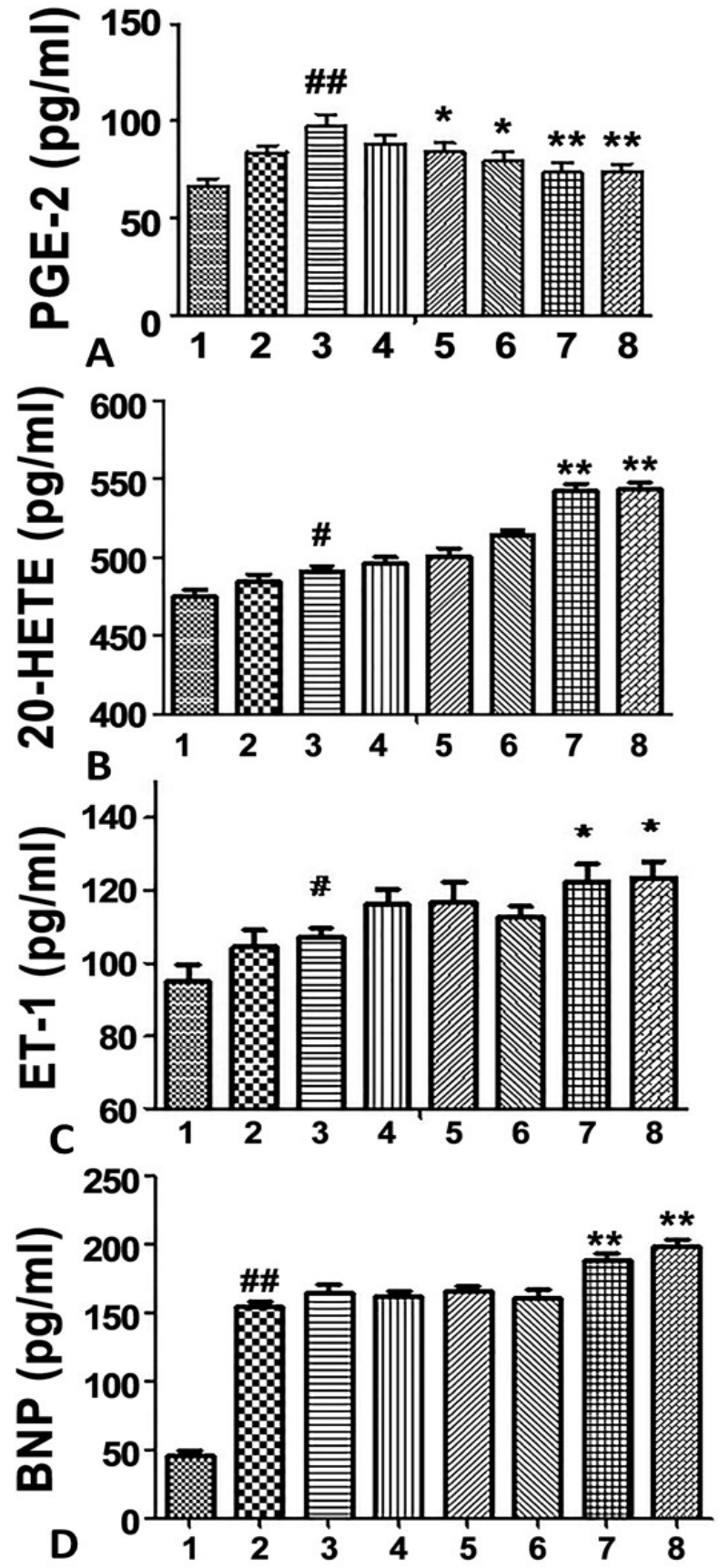

Fig. 3. Effects of drug-combination on the PGE-2 (A), 20-HETE (B), ET-1 (C) and BNP (D) levels in the experimental groups. Data are the mean $\pm \mathrm{SD} .{ }^{*} P<0.05$, ${ }^{\#} P<0.01$ compare with control group; ${ }^{*} P<0.05,{ }^{* *} P<0.01$ compare with model group. group $(P<0.05)$. Compared with the model group, the treatment group was increased, especially the combination medication group $(P<0.01)$.

\section{Effect of combination medication on BNP in the rat blood serum}

In our experiment, BNP concentration in the rats' blood serum is illustrated in Figure 3D. It shows that the model group displayed increased levels of BNP by comparing the relative data with the control group $(P<0.05)$. And treatment group all showed increase in comparison with the model group, especially the combination medication group $(P<0.01)$.

\section{Immunohistochemical results analysis}

From top to bottom the pictures of immunohistochemical results under the microscope were, in order, tissues of the heart, the liver and the kidney. As shown in Figure 4 CYP4A1 was detected in the heart tissues weakly. Notably, the expression of CYP4A1 in the rosiglitazone-treated group and combination group was increased compared with the control group. In liver tissues, CYP4A1 expression was seldom expressed, treatment with rosiglitazone and rofecoxib group showed a relative high expression. In the kidney tissues, CYP4A1 expression was high, which can be seen in the cytoplasm and cell membrane of the glomerulus. In addition, the expression levels of groups with medication treatment were above the data in the control group and model groups, while the combination group expressed highest levels.

\section{Effect of combination medication treatment on CYP4A1 protein levels}

To test the effect of drug combination on the expression level of CYP4A1 protein, we analyzed renal microsomes with western blot using polyclonal antibodies against CYP4A1/2/3 in rats. As shown in Figure 5, CYP4A1 expression in the kidney tissues was observed in each experimental group. Whereby, the expression of CYP4A1 in the rosiglitazone-treated group and combination medication group was increased compared with the control group. Notably, the expression of CYP4A1 was detected weakly in liver and heart tissues, and combination group shown a higher expression. 

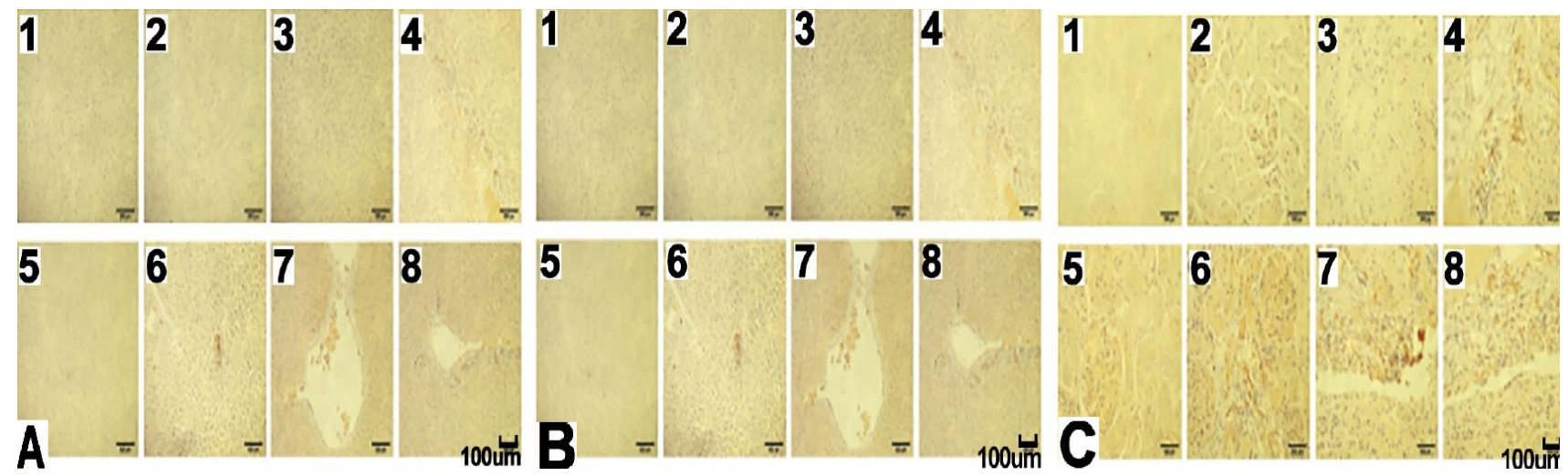

Fig. 4. Effects of drug unification on immunohistochemical. A. Heart tissues. B. Liver tissues. C. Kidney tissues. (1) Control group; (2) diabetes model group; (3) model group of combined disease; (4) rosiglitazone group; (5) rofecoxib group; (6) celecoxib group; (7) treatmented with rosiglitazone and rofecoxib group; (8) treatmented with rosiglitazone and celecoxib group. (Scale bars

$=100 \mathrm{um})$.

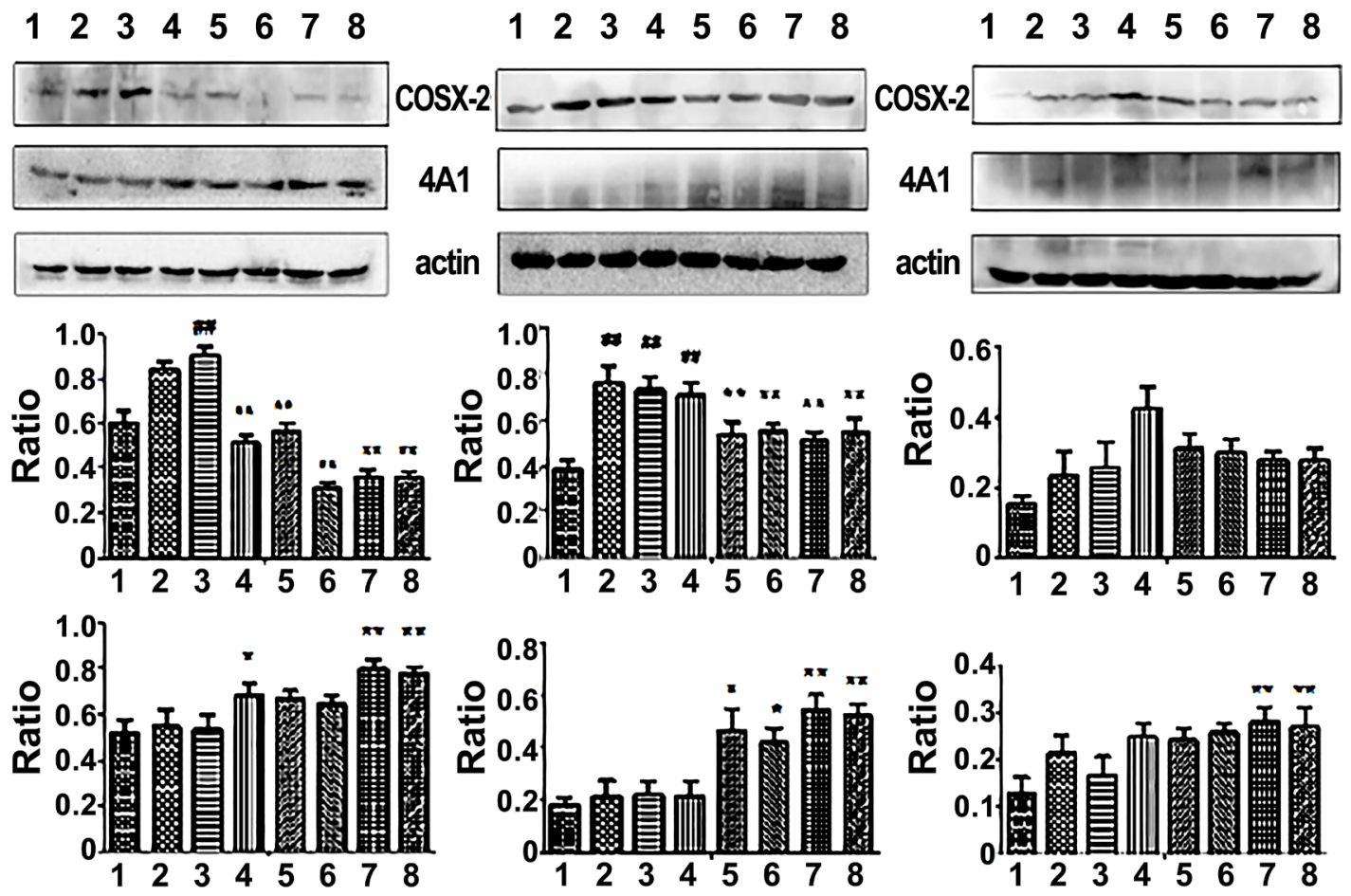

Fig. 5. Effect of drug combination on the protein of CYP4A1, COX-2 in the experimental groups (A) Kidney. (B) Heart. (C) Liver. Data are the mean \pm SD. ${ }^{\#} P<0.05,{ }^{\#} P<0.01$ compare with control group; ${ }^{*} P<0.05,{ }^{* *} P<0.01$ compare with model group.

\section{Effect of combination medication treatment on $\mathrm{COX}-2$}

The reaction led by $\mathrm{COX}-2$ is the core of inflammatory reaction, which may produces a large number of inflammatory mediators. After administrated COX-2 inhibitors, as shown in Figure 5, the expression of COX-2 was detected significantly in the model group. What's obvious is that COX-2 expression in the tissues considerably climbed in the model group $(P<0.01)$. Besides, the rosiglitazone-treated group displayed slightly decrease and anti-inflammatory group and combination medication group shown significant decrease in COX-2 expression $(P<0.01)$. 


\section{DISCUSSION}

In this experiment, we successfully established the model of diabetes complicating arthritis rats. Arachidonic acid involved in the inflammation by COX-2 enzyme, on experiment we observed that COX-2 inhibitors blocked the expression of COX-2, it resulted a reduced level of PGE2 in blood serum of inflammatory model group of rats. Nevertheless, owing to its specificity, the less effect on COX-1 may have little impact on TXA2, which may induce the imbalance of PGI2/TXA2. On the other hand, we observed that level of 20-HETE was increased in model group.

20-HETE is derived from arachidonic acid metabolized by CYP4A family in human body (Wang et al., 2015). And it is an eicosanoid that regulates a countless of actions in the vascular system (Hoopes et al., 2015). As a metabolite of arachidonic acid, 20-HETE has previously been reported that it can increase platelet aggregation and cause hemostasis (Wang et al., 2015). The up-regulates the expression of CYP4A was induced by rosiglitazone, a PPAR- $\gamma$ agonist, and it is reported that PPAR $-\gamma$ agonist has improved the transcriptional activity of PPAR- $\gamma$-CYP4A1 transfect cells (Juge-Aubry et al., 1997). From experimentation results, the expression of CYP4A1 in different tissues in rats showed that medicated group expressed higher than other groups, which proved this statement. Owing to the up-regulated expression of CYP4A1, the 20-HETE further generated ultimately results in higher blood pressure of rats. Meanwhile, COX-2 inhibitors blocked the first metabolic pathway of arachidonic acid, resulting in the rise of initial concentration. At the same time, more arachidonic acid was transformed into 20-HETE by the metabolism of CYP4A1. The current evidence presented by Liu et al. (2010) suggests that those rofecoxib acts, can inhabit the degradation of 20-HETE to some extent, which an effective vasoconstrictor, could increases the risk of stroke and myocardial infarction.

ET-1 is the primary member of the endothelin family and the most effective vasoconstrictor identified (Gebremedhin et al., 1992). Elevated ET-1 levels are associated with adverse cardiovascular effects (ValeroMunoz et al., 2016). BNP has been confirmed to be useful (Clerico and Emdin, 2004) for screening of heart disease (Vasan et al., 2002), stratification of patients with heart failure (HF) (Koglin et al., 2001; Bettencourt et al., 2004), detection of left ventricular systolic and/or diastolic dysfunction (Clerico and Emdin, 2004) and so on. Finally, it was reported that it is important to recognize that the two diseases (diabetic and hypertensive) always coexist and the cardiovascular disease are inextricably contacted with each other, the cardiovascular diseases are more possibly exist in this joint diseases (Sowers and Sowers, 1992).

Here we demonstrate the mechanism for increasing the combination of blood pressure. Selective COX-2 inhibitors excert less impact on COX-1 accompany with little impact on TXA2, which may inducing the imbalance of PGI2/TXA2. Rosiglitazone, a PPAR- $\gamma$ agonist, increased the CYP4A1 expression and the level of 20-HETE, which may caused blood vessel contraction and the rise of blood pressure. COX-2 inhibitors inhibit the degradation of 20HETE, which caused the increase of relative concentration and blood pressure. COX-2 inhibitors blocked the first metabolic pathway of arachidonic acid, resulting in the rise of initial concentration. Meanwhile, more arachidonic acid was transformed into 20-HETE by the metabolism of CYP4A1. ET-1 and BNP are the useful biomarkers in the progression of heart failure. Therefore, the increasing level of this two may imply the risk of heart failure in combination medication. As shown in Figure 6, here is the possible mechanism of combined therapy with COX-2 inhibitors and TZDs.

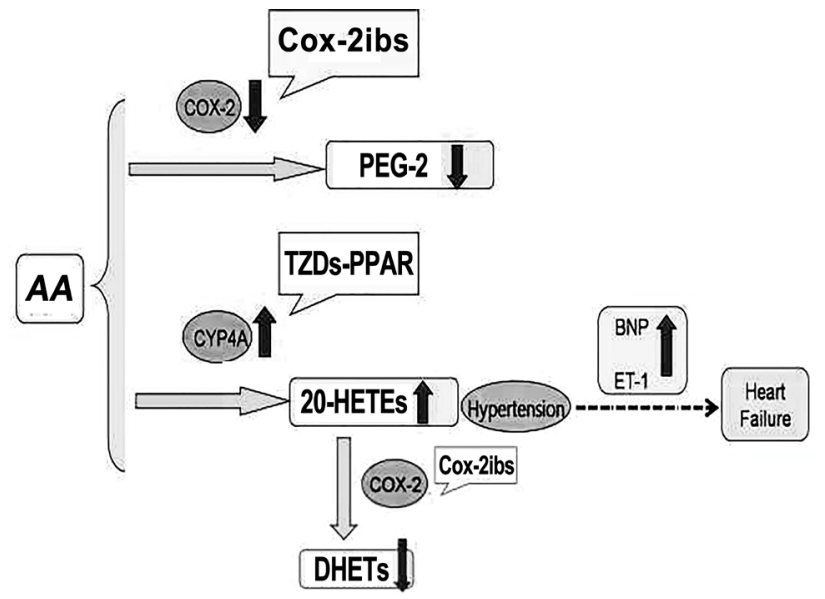

Fig. 6. Proposed mechanism of potential cardiovascular (cvs) side effects of combined therapy with Cox-2 inhibitors and TZDs.

Our study also has several limitations. In this experiment, only one of the factors in the large cardiovascular system-blood pressure was measured. Blood pressure acts as a major factor in the cardiovascular system and there were many unstable factors in the measurement of animals which made blood pressure collection work difficult and time-consuming, we only carried out measurement of blood pressure. In the further exploration, we will continue the research on the role of CYP4A1-20-HETE in cardiovascular function. In addition, the other cardiovascular indicators will be measured to perfect the research. We adopted rofecoxib which had 
been prohibited in the world. Because the side effect of rofecoxib on cardiovascular system is stronger and the mechanism is maturer, clearer and more representative, we can guarantee the controllability of the experiment and the use of rofecoxib was helpful to realize the experimental results. We only measured the endogenous 20-HETE catalyzed by the enzyme CYP4A1, while no experimental part of exogenous 20-HETE was carried out. So, we will add it in later experiments to further clarify the importance of 20-HETE in the cardiovascular system. Combined therapy which caused side effects may induced by inhibiting COX-2 pathway and activating CYP4A pathway while inhibiting the metabolism of 20-HETE. The potential cardiovascular side effects of rosiglitazone or rofecoxib were also found in some articles, in fact, they may only affect either COX-2 pathway or CYP4A pathway. The associated groups were too much and the experimental period may still a short time, so the detailed total grouping situation and some reflection may still need to improve in our next experimental planning. The results of our experiment were not so obvious and it need prolong administration.

\section{CONCLUSIONS}

The unification medication is clinical generally used but some questions to need to be solved exist. Cardiovascular side effects could increase by combined therapy of these two types of drugs, and it realized mainly through cytochrome P450 pathway of arachidonic acid metabolism. We may inspire from the animal experiments that combination of coxibs and TZDs for patient is disappointing, which was mainly due to the aggravating risk of CVD (cardiovascular disease). With the drug combination in the future, in order to ensure its effectiveness and safety, combined treatment with antidiabetic and anti-inflammatory in the diabetes complicating acute inflammation patients, the staff need to observation variations in human cardiovascular indicators in order to prevent and reduce the occurrence of ADRs.

\section{ACKNOWLEDGEMENT}

We acknowledge the financial supports of the Anhui Province Natural Science Foundation under Grant No. $1808085 \mathrm{MH} 284$.

\section{Statement of conflict of interest}

The authors have declared no conflicts of interest.

\section{REFERENCES}

Alzaid, A.A., 2010. Goodbye Avandia! Saudi med. J.,
31: 1301-1302.

Bettencourt, P., Azevedo, A., Pimenta, J., Friões, F., Ferreira, S. and Ferreira, A., 2004. N-terminalpro-brain natriuretic peptide predicts outcome after hospital discharge in heart failure patients. Circulation, 110: 2168-2174. https://doi. org/10.1161/01.CIR.0000144310.04433.BE

Barton, J.L., 2009. Patient preferences and satisfaction in the treatment of rheumatoid arthritis with biologic therapy. Patient Prefer Adher, 3: 335-344. https://doi.org/10.2147/PPA.S5835

Bax, M., Heemst, J.V., Huizinga, T.W.J. and Toes R.E.M., 2011. Genetics of rheumatoid arthritis: what have we learned? Immunogenetics, 63: 459466. https://doi.org/10.1007/s00251-011-0528-6

Campbell, W.B., Gebremedhin, D., Pratt, P.F. and Harder, D.R., 1996. Identification of epoxyeicosatrienoic acids as endothelium-derived hyperpolarizing factors. Circul Res., 78: 415-423. https://doi.org/10.1161/01.RES.78.3.415

Cheng, Y., 2002. Role of prostacyclin in the cardiovascular response to thromboxane A2. Science, 296: 539-541. https://doi.org/10.1126/ science. 1068711

Clerico, A. and Emdin, M., 2004. Diagnostic accuracy and prognostic relevance of the measurement of the cardiac natriuretic peptides: a review. Clin. Chem., 50: 33-50. https://doi.org/10.1373/ clinchem.2003.024760

Delea, T.E., Edelsberg, J.S., Hagiwara, M., Oster, G. and Phillips, L.S., 2004. Use of thiazolidinediones and risk of heart failure in people with type 2 diabetes: A retrospective cohort study. Diabetes Care, 27: 852-853. https://doi.org/10.2337/diacare.27.3.852

Desk, R., Williams, L. and Health, K., 2002. National cholesterol education program expert panel on detection, E. and A. treatment of high blood cholesterol in adults (adult treatment panel III). Third Report of the National Cholesterol Education Program (NCEP) Expert Panel on Detection, Evaluation, and Treatment of High Blood Cholesterol in Adults (Adult Treatment Panel III) final report. Circulation, 106: 3143-3421. https:// doi.org/10.1161/circ.106.25.3143

FitzGerald, G.A., 2004. Coxibs and cardiovascular disease. N. Engl. J. Med., 351: 1709-1711. https:// doi.org/10.1056/NEJMp048288

Gebremedhin, D., Ma, Y.H., Falck, J.R., Roman, R.J., VanRollins, M. and Harder, D.R., 1992. Mechanism of action of cerebral epoxyeicosatrienoic acids on cerebral arterial smooth muscle. Am. J. Physiol., 263: 519-525. https://doi.org/10.1152/ 
ajpheart.1992.263.2.H519

Harder, D.R., Campbell, W.B. and Roman, R.J., 1995. Role of cytochrome P-450 enzymes and metabolites of arachidonic acid in the control of vascular tone. J. Vasc. Res., 32: 79-92. https://doi. org/10.1159/000159080

Hoopes, S.L., Garcia, V., Edin, M.L., Schwartzman, M.L. and Zeldin, D.C., 2015. Vascular actions of 20-HETE. Prostagl. Other Lipid Mediat., 120: 9-16. https://doi.org/10.1016/j. prostaglandins.2015.03.002

Imig, J.D., 2012. Epoxides and soluble epoxide hydrolase in cardiovascular physiology. Physiol. Rev., 92: 101-130. https://doi.org/10.1152/ physrev.00021.2011

Juge-Aubry, C., Pernin, A., Favez, T., Burger, A.G., Wahli, W., Meier, C.A. and Desvergne, B., 1997. DNA binding properties of peroxisome proliferator-activated receptor subtypes on various natural peroxisome proliferator response elements. Importance of the 5'-flanking region. J. biol. Chem., 272: 25252-25259. https://doi.org/10.1074/ jbc. 272.40 .25252

Kenneth, W.M., Hafley, G., Dickerson, S., Burns, S., Tourt-Uhlig, S., White, J., Newby, L.K., Komajda, M., McMurray, J., Bigelow, R., Home P.D. and Lopes, R.D., 2013. Results of a reevaluation of cardiovascular outcomes in the RECORD trial. Am. Heart J., 166: 240-249. https://doi.org/10.1016/j. ahj.2013.05.004

Koglin, J., Pehlivanli, S., Schwaiblmair, M., Vogeser, M., Cremer, P. and VonScheidt, W., 2001. Role of brain natriuretic peptide in risk stratification of patients with congestive heart failure. J. Am. Col. Cardiol., 38: 1934-1941. https://doi.org/10.1016/ S0735-1097(01)01672-2

Kroetz, D.L. and Zeldin, D.C., 2002. Cytochrome P450 pathways of arachidonic acid metabolism. Curr. Opin. Lipidol., 13: 273-283. https://doi. org/10.1097/00041433-200206000-00007

Liu, J.Y., Li, N., Yang, J., Qiu, H.,Ai, D., Chiamvimonvat, N., Zhu, Y. and Hammock, B.D., 2010. Metabolic profiling of murine plasma reveals an unexpected biomarker in rofecoxib-mediated cardiovascular events. Proc. natl. Acad. Sci., 107: 17017-17022. https://doi.org/10.1073/pnas.1011278107

Lukaszewicz, K.M., Falck, J.R., Manthati, V.L. and Lombard, J.H., 2013. Introgression of Brown Norway CYP4A genes on to the Dahl salt-sensitive background restores vascular function in SS-5 (BN) consomic rats. Clin. Sci. (Lond), 124: 333342. https://doi.org/10.1042/CS20120232
Mukherjee, D., Nissen, S.E. and Topol, E.J., 2001. Topol, Risk of cardiovascular events associated with selective COX-2 inhibitors. J. Am. med. Assoc., 286: 954-959. https://doi.org/10.1001/ jama.286.8.954

Nithipatikom, K., DiCamelli, R.F. and Kohler, S., 2001. Determination of cytochrome P450 metabolites of arachidonic acid in coronary venous plasma during ischemia and reperfusion in dogs. Anal. Biochem., 292: 115-124. https://doi.org/10.1006/ abio.2001.5044

Pfister, S.L. and Campbell, W.B., 1992. Arachidonic acid and acetylcholine-induced relaxations of rabbit aorta. Hypertension, 20: 682-689. https:// doi.org/10.1161/01.HYP.20.5.682

Ralph A.D., 2009. Banting Lecture. From the triumvirate to the ominous octet: A new paradigm for the treatment of type 2 diabetes mellitus. Diabetes, 58: 773-795. https://doi.org/10.2337/db09-9028

Ray, W.A., Griffin, M.R. and Stein, C.M., 2004. Cardiovascular toxicity of valdecoxib. N. Engl. J. Med., 351: 2767. https://doi.org/10.1056/ NEJMc045711

Reddy, K.K., Rajan, V.K., Gupta, A., Aparoy, P. and Reddanna, P., 2015. Exploration of binding site pattern in arachidonic acid metabolizing enzymes, Cyclooxygenases and Lipoxygenases. BMC Res. Notes, 16: 152. https://doi.org/10.1186/s13104015-1101-4

Roberto G., Piero R., Saverio A., Francesco C., Vasiliki L., Paola D. B., Giuliana G., Onorina B., Francesco C., Giovanni T. and Paola C., 2016. IL-1beta at the crossroad between rheumatoid arthritis and type 2 diabetes: may we kill two birds with one stone? Expert Rev. Clin. Immunol., 12: 849-855. https:// doi.org/10.1586/1744666X.2016.1168293

Sundy, J.S., 2001. COX-2 inhibitors in rheumatoid arthritis. Curr. Rheumatol. Rep., 3: 86-91. https:// doi.org/10.1007/s11926-001-0055-9

Sowers, K.M.R. and Sowers, J.R., 1992. Diabetes and hypertension. Hypertension, 19: 403-418. https:// doi.org/10.1161/01.HYP.19.5.403

Valero-Munoz, M., Li, S., Wilson, R.M., Boldbaatar, B., Iglarz, M. and Sam, F., 2016. Dual endothelin-A/ endothelin-B receptor blockade and cardiac remodeling in heart failure with preserved ejection fraction. Circ. Heart Fail., 9: e003381. https://doi. org/10.1161/CIRCHEARTFAILURE.116.003381

Varga, T., Czimmerer, L. and Nagy, L., 2011. PPARs are a unique set of fatty acid regulated transcription factors controlling both lipid metabolism and inflammation. Biochim. biophys. Acta, 1812: 1007- 
1022. https://doi.org/10.1016/j.bbadis.2011.02.014

Vasan, R.S., Benjamin, E.J., Larson, M.G., Leip, E.P., Wang, T.J., Wilson, P. and Levy, D., 2002. Plasma natriuretic peptides for community screening for left ventricular hypertrophy and systolic dysfunction. J. Am. med. Assoc., 288: 1252-1259. https://doi.org/10.1001/jama.288.10.1252

Wang, J.X., Li, H., He, J., Li, B., Bao, Q., Zhang, X., Lv, Z., Zhang, Y., Han, J., Ai, D. and Zhu, Y., 2015. 20-Hydroxyeicosatetraenoic acid involved in endothelial activation and thrombosis. Am. J. Physiol. Heart Circ. Physiol., 308: 1359-1367. https://doi.org/10.1152/ajpheart.00802.2014

Weerasinghe, G.R., Rapoport, S.I. and Bosetti, F., 2004. The effect of chronic lithium on arachidonic acid release and metabolism in rat brain does not involve secretory phospholipase A (2) or lipoxygenase/cytochrome P450 pathways. Brain Res. Bull., 63: 485-489. https://doi.org/10.1016/j. brainresbull.2004.04.005

Yasmin, S. and Jayaprakash, V., 2017. Thiazolidinediones and PPAR orchestra as antidiabetic agents: From past to present. Eur. J. med. Chem., 126: 879-893. https://doi.org/10.1016/j.ejmech.2016.12.020

Zhang, Y., Hoda, N.M., Zheng, X., Li, W.G., Luo, P.C., Maddipati, K.R., Seki, T., Ergul, A. and Wang, M.H., 2014. Combined therapy with COX2 inhibitor and 20-HETE inhibitor reduces colon tumor growth and the adverse effects of ischemic stroke associated with COX-2 inhibition. Am. J. Physiol. Regul. Integr. Comp. Physiol., 307: 693703. https://doi.org/10.1152/ajpregu.00422.2013 